Military Technical College Kobry El-Kobbah, Cairo, Egypt

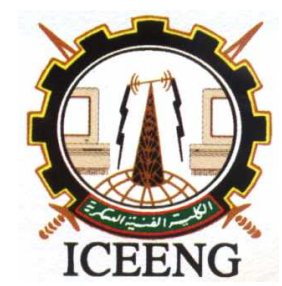

\author{
$6^{\text {th }}$ International Conference \\ on Electrical Engineering \\ ICEENG 2008
}

\title{
Modeling the Modified Ionosphere Parameters due to HF Heating Facilities
}

\author{
By \\ Mohamed A H Eleiwa (Ph.D.),*
}

\section{Abstract:}

A diagnostic algorithm is proposed to derive and model the ionosphere parameters modified by powerful high frequency (HF) radio waves. The proposed model is composed of three steps: characterization of the HF ionosphere heaters in terms of location, effective radiated power, frequency, and operation modes parameters, diagnostic of the ambient ionosphere parameters, and simulation of the effects of the heating experiment on the ionosphere. The proposed algorithm is applied to investigate the artificial ionospheric irregularities and instabilities produced by HF heating experiments reported from different ground-based HF heating facilities such as HAARP at Alaska, and EISCAT heating facility in Tromso. The ionospheric instability and irregularity parameters are then derived from the measured modified ionospheric parameters during heating experiments. The conditions for the hydrodynamical equilibrium disruption and initiation of the artificial aurora are derived and discussed in terms of the HF heating facilities parameters.

\section{Keywords:}

Ionosphere, High power EM waves, Ionosphere HF Heating facilities 


\section{Introduction:}

The ionosphere [1] is an example of naturally occurring plasma formed by solar photo-ionization. Ultraviolet and $\mathrm{x}$-ray radiations from the sun ionize the atmospheric constituents. In the ionosphere, a balance between photo-ionization and various loss mechanisms gives rise to an equilibrium density of free electrons and ions with a horizontal stratified structure. The density of these electrons is a function of the height above the earth's surface and is affected by the sunrise and sunset, especially at the lower altitudes. The presence of these free electrons in the ionosphere can influence the propagation of radio waves. The ionosphere is conventionally divided into the D-, E-, and F-regions. The D-region lies between 60 and $95 \mathrm{~km}$, the E-region between 95 and $150 \mathrm{~km}$, and the F-region lies above $150 \mathrm{~km}$. In daylight, it is possible to distinguish two separate layers within the F-region, the F1 (lower) and the F2 (upper) layers. During nighttime, these two layers combine into one single layer. The electron distribution throughout these layers is subject to diurnal, daily, seasonal, and geographical variations as well as the magnetic disturbance associates with solar activities.

Different methods have been explored to artificially modify the ionosphere including injection of chemical vapors and heating or charging via electromagnetic radiation or particle beams [2]. It is important to note that many techniques to modify the upper atmosphere have been successfully demonstrated experimentally. Ground based modification techniques include vertical and oblique HF heating, microwave heating, and magnetospheric modification. Several significant applications of such ionosphere modifications techniques include very and low frequency (VLF, LF) communications, HF ducted communications, and creation of artificial ionospheric mirrors (AIM) for pinpoint communication or over-the-horizon (OTH) radar transmission.

Ionospheric heating facilities with powerful, ground-based HF transmitters have a broad spectrum of research goals. One direction of this research is to study how the heating modifies parameters of the ionosphere itself, including modification of the plasma density and temperature in the ionospheric layers at various spatial and temporal scales. Another direction combines studies of how the modification of the ionosphere with HF heating affects propagation of electromagnetic (EM) waves through the heated region. A third direction includes studies of how heating the ionosphere in the electrojet regions can generate electromagnetic waves propagating from the ionosphere to the ground. A fourth direction involves studies of EM waves generated by the heating, which propagate to the magnetosphere. Extensive overviews of the ongoing research activities in these areas are given in recent papers [3-8].

This paper focuses on the analysis of the ionospheric heating experiments performed to artificially modify the parameters of the ionosphere, and thence the assessment of controlling the artificial ionospheric irregularities and instabilities using 
such heating facilities. The resulting artificial irregularities parameters are derived in terms of the heating facility parameters. The ionospheric parameters due to natural and artificial modification techniques are also presented, studied, and compared. To estimate the artificial ionosphere disruption effects. First, the variation of quite plasma parameters $\left(T_{e}, T_{i}\right.$, and $T_{n}$, electron, ion, and neutral plasma temperatures, and also the ionosphere density constituents $\left.\mathrm{N}\left(\mathrm{N}_{2}\right), \mathrm{N}\left(\mathrm{O}_{2}\right), \mathrm{N}(\mathrm{O}), \mathrm{N}(\mathrm{He}), \mathrm{N}_{\mathrm{e}}, \mathrm{N}_{\mathrm{i}}\right)$ is studied versus time, altitude, latitude, and longitude at the ambient ionosphere without heating. The heating facility parameters (geographical location, frequency $\mathrm{f}$, effective radiated power ERP, and the operation mode) are input to the proposed code. Then, the modified ionospheric parameters during heating experiments are derived and compared with the corresponding quite plasma parameters, and thence the artificial irregularity parameters of the disrupted ionosphere are derived, analyzed, compared, and discussed in terms of the heating facility parameters. The interaction of the ionosphere on impinging powerful $\mathrm{HF}$ radio waves is analyzed, and hence the possible mechanisms of the artificial irregularities and instabilities induced in the ionosphere by ground-based HF heating facilities are modeled in section II. The measured parameters and capabilities of several ionospheric heating facilities experiments are simulated, analyzed and compared in section III. Then, the ionospheric instability and irregularity parameters are derived, compared, and discussed in terms of the heating facility parameters. Hence the effects of different heating experiments on the ionospheric medium and wave parameters are assessed. Finally, discussions and conclusions are presented in section IV.

\section{Electromagnetic waves-Ionosphere Interaction Model.:}

The homogeneous imperfectly conducting medium is characterized by the complex dielectric constant (relative permittivity; $\boldsymbol{\varepsilon}_{\boldsymbol{c}}$ ), which may be written as [9]

$\sqrt{\varepsilon_{\mathrm{c}}^{\prime}}=\sqrt{\frac{\varepsilon_{\mathrm{c}}}{\varepsilon_{\mathrm{o}}}}=\sqrt{\varepsilon^{\prime}-\mathbf{j} 60 \sigma \lambda}=\mathbf{n}-\mathbf{j p}$

Solving the complex equation (1) to yield its real and imaginary parts as

$$
\begin{aligned}
& \mathbf{n}= \pm \sqrt{\mathbf{0 . 5}\left(\varepsilon^{\prime}+\sqrt{\left(\varepsilon^{\prime}\right)^{2}+(60 \sigma \lambda)^{2}}\right)} \\
& \left.\mathbf{p}= \pm \sqrt{\mathbf{0 . 5}\left(-\varepsilon^{\prime}+\sqrt{\left(\varepsilon^{\prime}\right)^{2}+(60 \sigma \lambda)^{2}}\right.}\right)
\end{aligned}
$$

The homogeneous ionized gas (ionosphere) behaves like an imperfectly conducting medium with conductivity $\sigma_{i}$ and dielectric constant $\varepsilon_{i}$ which are given by [10] as

$$
\varepsilon_{i}^{\prime}=1-\frac{\omega_{p}^{2}}{\omega^{2}+v^{2}}
$$


$\sigma_{i}=\frac{\varepsilon_{0} \omega_{p}^{2} v}{\omega^{2}+v^{2}}$

Where $\omega=\mathbf{2} \pi \mathbf{f}$ is the propagating wave frequency, $\boldsymbol{v}$ is the collision frequency, $\omega_{\mathrm{p}}=\sqrt{\frac{\mathbf{N}_{\mathrm{e}} \mathbf{q}_{\mathrm{e}}^{\mathbf{2}}}{\mathbf{m}_{\mathrm{e}} \varepsilon_{\mathrm{o}}}}$ is the plasma frequency, $\boldsymbol{N}_{e}$ is the density of free electrons, $\boldsymbol{m}_{e}$, and $\boldsymbol{q}_{e}$ are mass and charge of the electron. The phase velocity of the propagating wave is $v_{p h}=c / n$ where $\boldsymbol{c}$ is the light speed and $\boldsymbol{n}$ is the refractive index of equation (2). As the radio waves travel through an imperfectly conducting medium, such as the ionosphere, short waves experience attenuation due to the absorption of energy. The extent of attenuation is characterized by the exponential form $\boldsymbol{e}^{-a l}$ where $\boldsymbol{l}$ is the distance travelled by the wave, $\alpha=\frac{\mathbf{p} \omega}{\mathbf{c}}$ is the absorption (attenuation) coefficient, and $\boldsymbol{p}$ is given by equation (3). In the presence of terrestrial magnetic field $\left(\boldsymbol{H}_{\boldsymbol{o}}\right)$, the electron rotates and exhibits resonance characteristics called gyro magnetic resonance with gyro frequency $\mathbf{f}_{\mathrm{m}}=\mathbf{q}_{\mathrm{e}} \boldsymbol{\mu}_{\mathbf{o}} \mathbf{H}_{\mathbf{o}} /\left(\mathbf{2} \pi \mathbf{m}_{\mathrm{e}}\right)$. The real ionosphere is an inhomogeneous ionized gas, and is assumed to consist of thin plane strata within each of which the electron number density $N_{e}(\boldsymbol{h})$ is constant, but depending on the height $\boldsymbol{h}$ of the assumed thin ionosphere layer. Accordingly, the plasma frequency $\omega_{p}$, and the other ionosphere parameters given by the above equations are all functions of height. Knowledge of the ionospheric electron density profile is essential for a wide range of applications. Several models have been explored to find $N_{e}$ for natural ionosphere such as the phenomenological Chiu model [11], the Bent model [12], the semi-empirical SLIM [13] and the IRI models [14]. Since the enhancement regions are not predicted by such models alone, therefore in this paper some of these models (MSIS-E and IRI) are only used to estimate numerically the undisturbed ionosphere parameters. Different parametric models are then derived from the estimated and measured data.

Several theories have been developed to explain the mechanism of artificial aurora initiation due to the interaction of a powerful electromagnetic wave with the ionosphere. According to conventional breakdown (CB) theory [15], the generated sustained intense electric fields heat and ionize the background medium until the aurora is initiated. Alternatively, the runaway theory [16] introduces another breakdown model, in which the preexisting high energy electrons are heated by the same generated sustained intense electric fields, leading to the formation of an electron beam. The collisions between this electron beam and the air molecules result in optical emission. On the other hand, the modified ionosphere affects the heating EM waves. As a result, the whole system becomes a complicated nonlinear and time-variant system. The resulting nonlinear heating process parameters: collision, ionization and recombination coefficients are 
included in the proposed model. The electron collision frequency $\boldsymbol{v}_{\boldsymbol{e}}$ may be derived from the equation of electron motion under the effect of electric field $\boldsymbol{E}$ as

$v_{\mathrm{e}}=\frac{\mathbf{q}_{\mathrm{e}}}{\mu_{\mathrm{e}} \mathbf{m}_{\mathrm{e}}}$

Where the electron mobility $\boldsymbol{\mu}_{\boldsymbol{e}}$ is inversely proportional to $\boldsymbol{E}$ by the drift velocity factor.

The other nonlinear process, ionization, is described by [17] as

$$
\frac{\mathbf{d} \mathbf{N}_{\mathrm{e}}}{\mathbf{d t}}=\left(\mathrm{v}_{\mathrm{i}}-\mathrm{v}_{\mathrm{a}}\right) \mathbf{N}_{\mathrm{e}}
$$

Where the ionization $\left(v_{i}\right)$ and attachment $\left(v_{\mathbf{a}}\right)$ coefficients are defined as

$v_{i}=5.1 \times 10^{-13} N\left(\frac{E}{E_{b d}}\right)^{2}\left(1+6.3 e^{-2.6 E_{b d} / E}\right) e^{-4.7 E_{b d} /\left(E_{b d}-E\right)}$

$v_{\mathrm{a}}=\sum_{\mathrm{i}=\mathbf{0}}^{2} \mathbf{a}_{\mathrm{i}} \mathbf{x}, \quad$ when $\quad \mathbf{x} \geq 1.62 \times 10^{6} \mathrm{~V} / \mathrm{m}$

$\log \left(\frac{\mathrm{v}_{\mathrm{a}} \mathbf{N}_{\mathrm{o}}}{\mathrm{N}}\right)=\sum_{\mathrm{i}=\mathbf{0}}^{3} \mathbf{b}_{\mathrm{i}}(\log \mathbf{x})^{2}, \quad$ when $\quad \mathbf{x} \geq 1.62 \times 10^{6} \mathrm{~V} / \mathbf{m}$

Where $x=E N_{o} / N, a_{o}=-2.41 x 10^{8}, a_{1}=211.92, a_{3}=-3.545 \times 10^{-5}, b_{o}=1073.8, b_{1}=$ 465.99, $b_{2}=-66.867$, and $b_{3}=3.197$.

\section{Simulation of the Ionosphere Modification by HF Heating Facilities:}

A simulation model is introduced to explain and compare the results of artificial ionospheric modification due to several heating experiments performed by different ground-based heating facilities. The proposed model includes characterization of the heating facilities parameters from the experimental observations using the equations derived in section II. Heating effects on the ionosphere parameters are then evaluated, and hence the ionospheric modification capabilities of different heating facilities are assessed and compared. The conditions for ionosphere hydrodynamical equilibrium disruption and initiation of artificial aurora events are also derived and discussed.

The proposed model is first used to evaluate the high frequency active auroral research program (HAARP), which is located in Alaska at $\mathbf{6 2 . 0 9}^{\circ}$ geomagnetic latitude, and its technical parameters are given in Table 1 . The simulation results from the numerical code developed by [18] are regenerated and plotted vs. ionosphere altitude in Fig.1, where the 
normalized attachment coefficients $\left(\boldsymbol{v}_{\boldsymbol{a}}\right)$ and the ambient electron temperature $\left(\boldsymbol{T}_{\boldsymbol{e}}\right)$ are compared with the electron temperatures $\left(\boldsymbol{T}_{\boldsymbol{e}}\right)$ due to ionospheric heating with HAARP EM waves having frequency $\boldsymbol{f}=\mathbf{9} \mathbf{M H z}, X$-mode polarization, and the effective radiated powers are 83.4 $d B W$ and $95.7 d B W$.

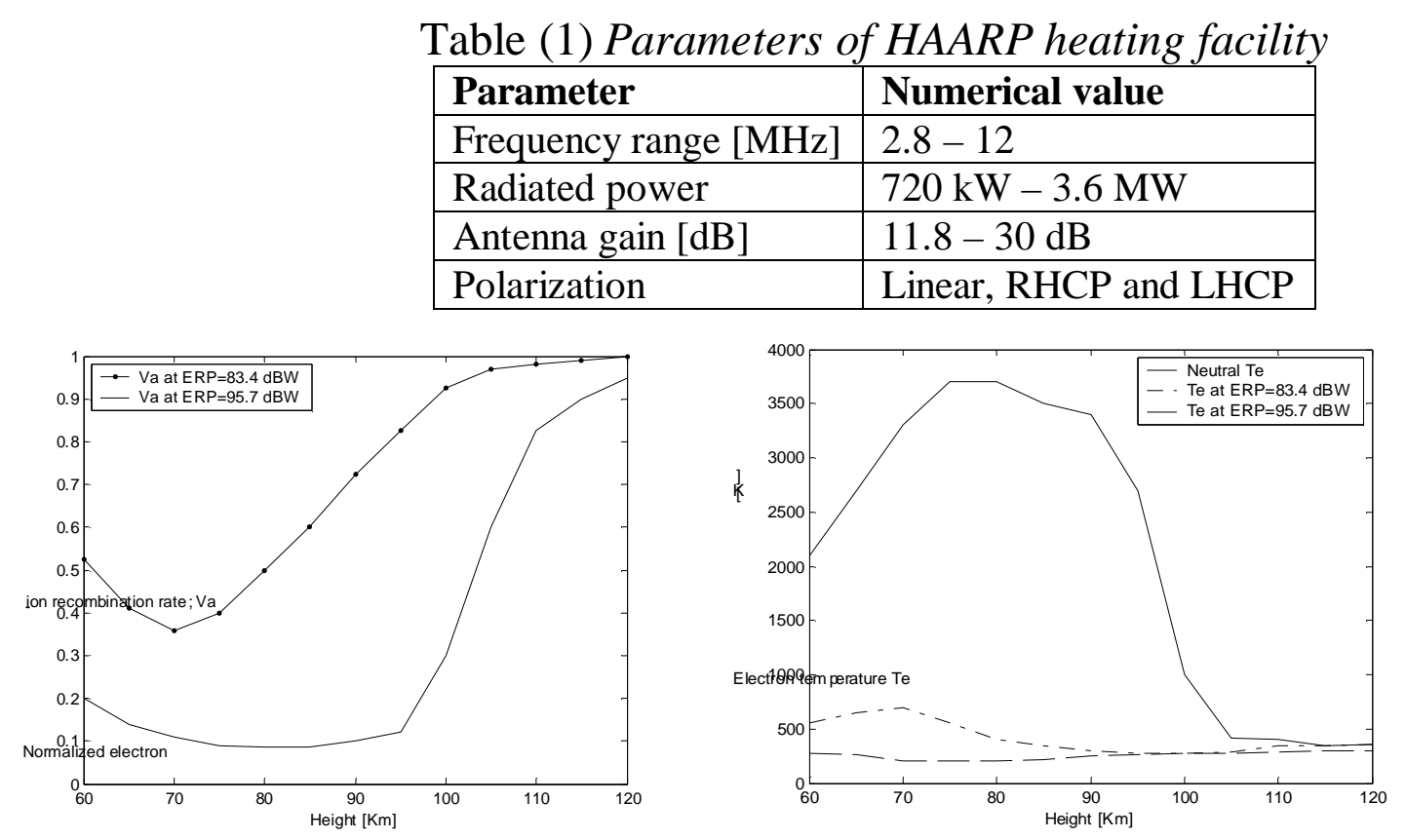

Figure(1): Variation of the attachment coefficients $\left(\boldsymbol{v}_{\boldsymbol{a}}\right)$ and the electron temperature $\left(\boldsymbol{T}_{\boldsymbol{e}}\right) v \boldsymbol{s}$. height of the ionosphere above HAARP facility at Alaska

It is clear from Fig. 1 that the normalized attachment (electron-ion recombination) coefficient is generally decreased with injected power increase leading to electron density increase, while the electron temperature $\left(\boldsymbol{T}_{\boldsymbol{e}}\right)$ is dramatically increased for higher injected radiated power, where $\boldsymbol{T}_{\boldsymbol{e}}$ exceeds $3500 \mathrm{~K}$ at $75 \mathrm{~km}$ height and the injected effective radiated power is $E R P=95.7 d B W$, compared to $500 K$ at $E R P=83.4 d B W$, and $200 K$ for undisturbed ionosphere. Further, the electron temperature variation vs. height is investigated in the ionosphere E region above HAARP location, as shown in Fig.2. 

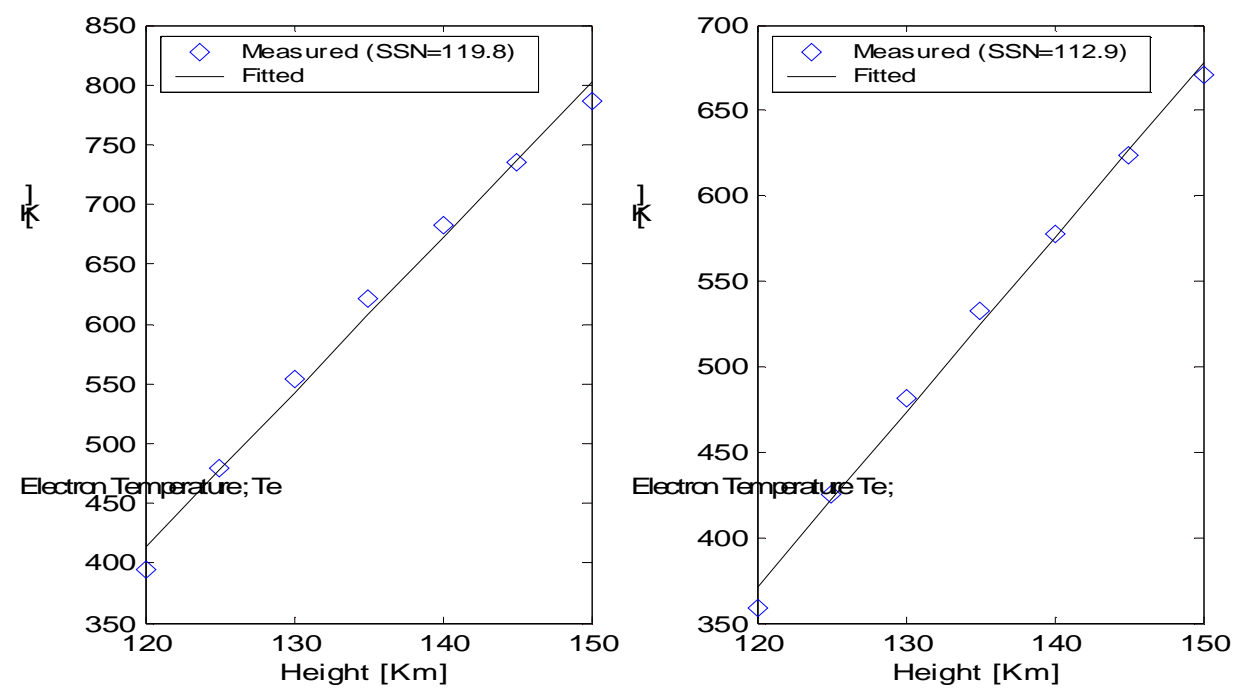

Figure(2): Temperature variations vs. height for different solar flux numbers (SSN)

Where the measured data obtained by IRI and MSIS models are fitted with different polynomials at different solar activities. The left curve is fitted with the polynomial $T_{e}(h)=13 h-1143.6$, for $S S N=119.8$ on $01 / 08 / 2000$ at 09.00 local time (LT), and the right one is fitted with the polynomial $T_{e}(\boldsymbol{h})=10.2143 \boldsymbol{h}-854.357$, for $S S N=112.9$ on $01 / 01 / 2000$ at 20.00 local time (LT). The fitted polynomials for the corresponding electron density $\left(N_{e}\right)$ profiles are derived, plotted and compared with the measured data as shown in Fig.3
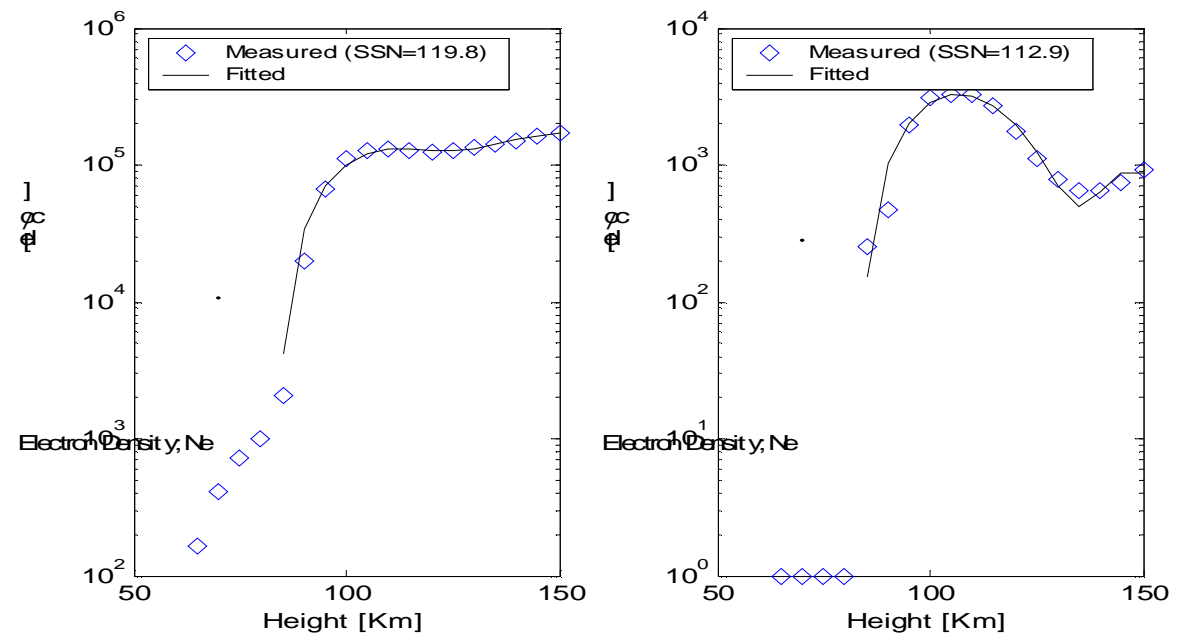

Figure(3): Electron density variations vs. height for different solar flux density numbers (SSN)

The derived polynomials describing the electron density and temperature variations in the ionosphere $\boldsymbol{D}$ and $\boldsymbol{E}$ regions above HAARP at Alaska in USA at different solar activity events in winter and summer at different local times are plotted and compared in Fig.4. 

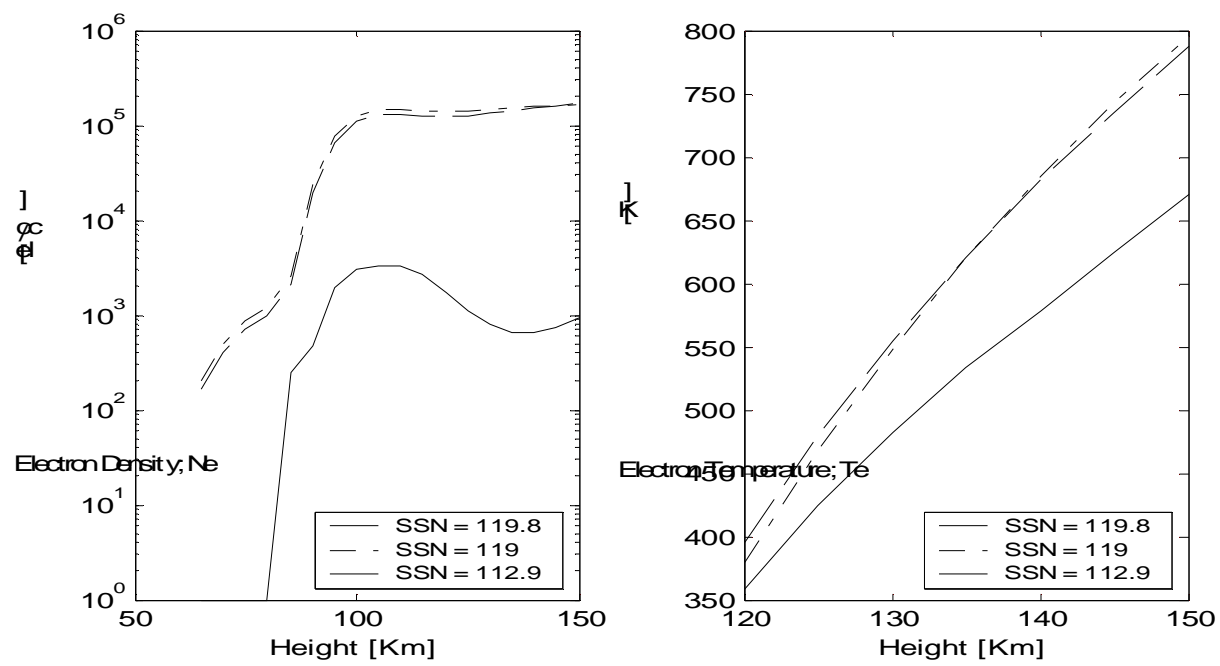

Figure(4): Electron density and temperature variations vs. height for different solar flux numbers (SSN)

From the derived polynomials shown in Fig.4 for electron temperature and density variations vs. height at different solar activity events, a general relation describing the average variation of $N_{e}$ with $\boldsymbol{T}_{\boldsymbol{e}}$ is derived and used to estimate the ionosphere hydrodynamical equilibrium disruption coefficient (EqDC). The condition of the hydrodynamical equilibrium is fulfilled when the relative plasma partial pressure is kept constant. The relative ionosphere partial pressure is given by [19] as

$$
\frac{\mathbf{P}}{\mathbf{P}_{\mathrm{o}}}=\frac{\mathbf{N}_{\mathrm{e}}\left(\mathbf{T}_{\mathrm{e}}+\mathbf{T}_{\mathrm{i}}\right)}{\mathbf{N}_{\mathrm{eo}}\left(\mathbf{T}_{\mathrm{e} o}+\mathbf{T}_{\mathrm{io}}\right)}
$$

Where $\boldsymbol{T}_{\boldsymbol{e} \boldsymbol{o}}, \boldsymbol{T}_{\boldsymbol{i o}}, \boldsymbol{N}_{\boldsymbol{e} \boldsymbol{o}}$ are the electron and ion temperatures and the electron density before the HF heater is turned on, while $\boldsymbol{T}_{\boldsymbol{e}}, \boldsymbol{T}_{\boldsymbol{i}}, \boldsymbol{N}_{\boldsymbol{e}}$ are the same parameters in the course of the heateron periods. The ionosphere equilibrium disruption coefficient (EqDC) is calculated as the normalized relative plasma partial pressure and plotted in Fig.5 for different injected ERP powers into the ionosphere above HAARP facility. 

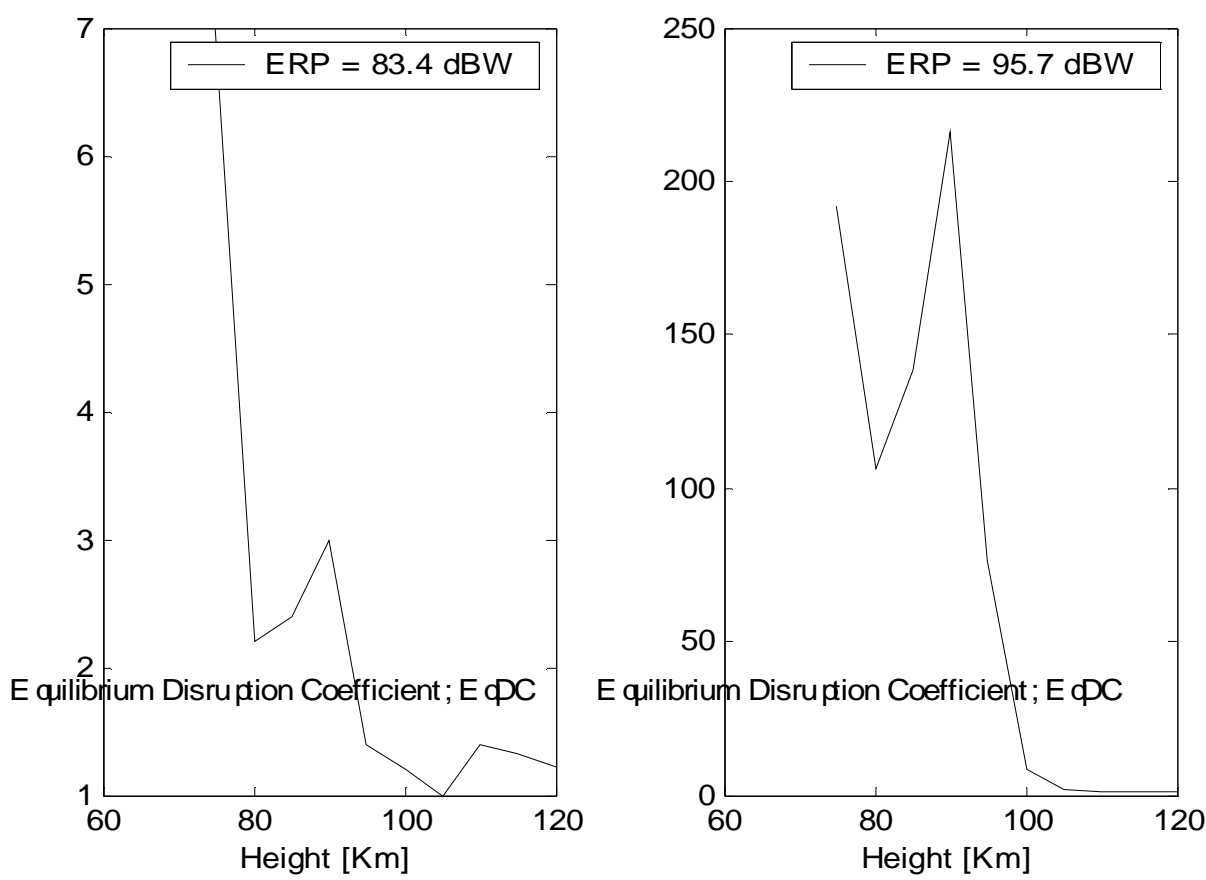

Figure(5): Ionosphere equilibrium disruption coefficients due to HAARP heaters with different pumping powers

It is clear from Fig.5 that the hydrodynamical equilibrium of the ionosphere is badly disrupted at heights up to $\mathbf{8 0} \mathbf{~} \mathbf{~ m}$ where the equilibrium disruption coefficient is greater than 5 for $\boldsymbol{E R P}=83.4 \mathrm{dBW}$, while the ionosphere hydrdynamical equilibrium is severely disrupted at heights up to $100 \mathbf{~} \mathbf{m}$ when the injected power is increased to $\boldsymbol{E R P}=\mathbf{9 5 . 7} \mathbf{d B W}$. The HF heating experiments of EISCAT facility in Troms $\emptyset$ is also analyzed. The experiment reported here was conducted by [20] using the EISCAT HF heating facility located near Troms $\varnothing$ (geographical coordinates 69:6_N, 19:2_E) in the pre-midnight hours of February 16, 1996. The Troms $\varnothing$ heater was operating at the frequency $4040 \mathbf{k H z}, \boldsymbol{O}$-mode polarization, with an effective radiated power of $150 \mathrm{MW}$. The antenna beam was tilted $\boldsymbol{6}^{\circ}$ to the south, thus allowing HF pumping in a near-field-aligned direction. The HF pumping experiment started at $2100 \mathrm{UT}$. The pump wave was modulated with a $4 \mathrm{~min}$. on, $6 \mathrm{~min}$. off cycle from 2100 to $2300 \mathrm{UT}$. This was preceded by some short on periods as the HF transmitter was tuned, from 2041 to 2044 UT, from 2046:30 to 2048:30 UT, and from 2052:00 to 2052:20 UT.

The experimental results (electron and ion temperatures; $\boldsymbol{T}_{\boldsymbol{e}}$ and $\boldsymbol{T}_{\boldsymbol{i}}$ respectively) obtained at altitude $\boldsymbol{h}=150 \mathrm{~km}$ on $\boldsymbol{F e b} .16,1996$ from 2030 to 2300 UT are displayed and compared with their corresponding parameters for unheated ionosphere in Fig.6. 

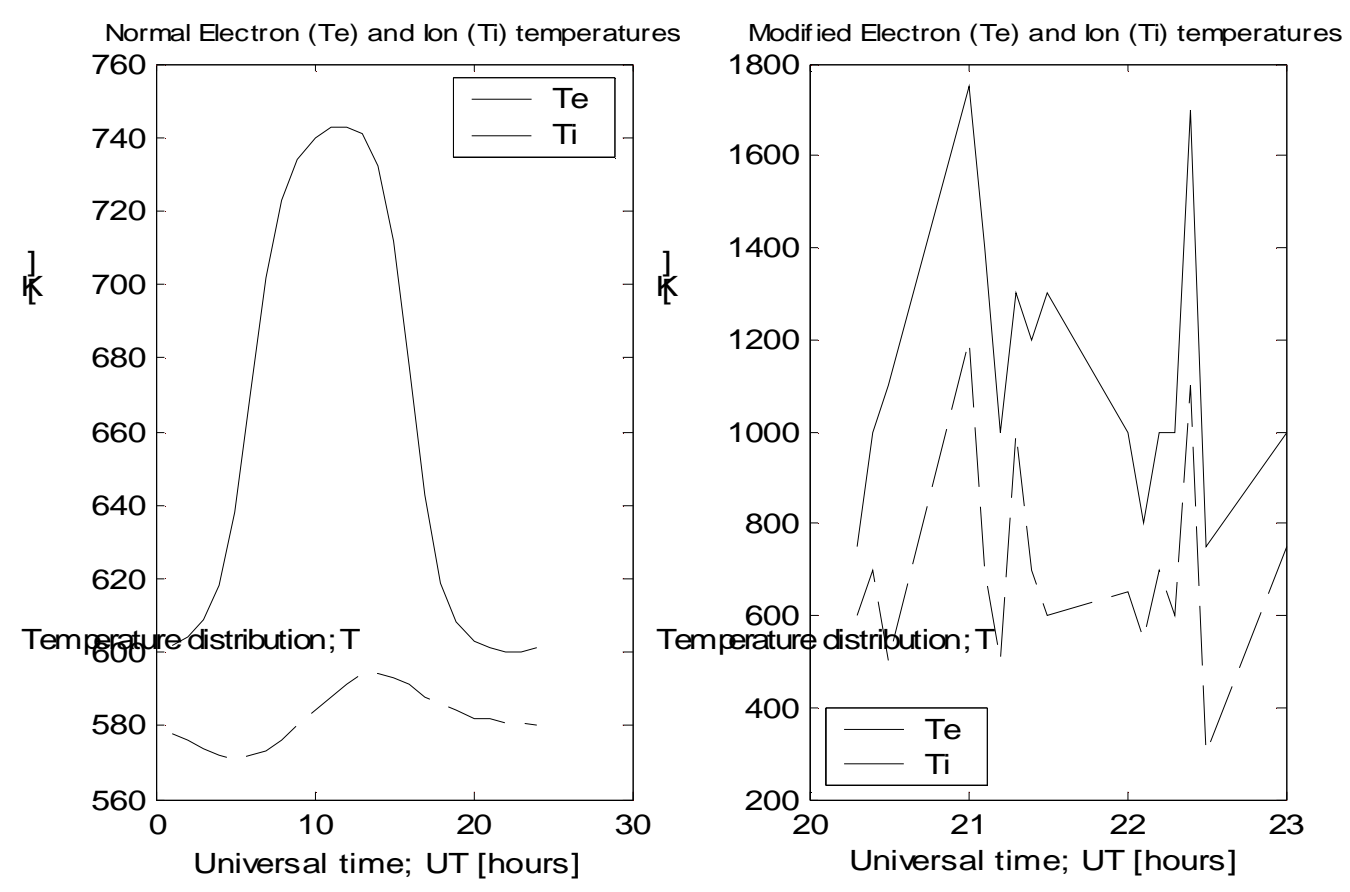

Figure(6): Variation of normal and modified electron and ion temperatures vs. universal time (UT) at $\mathbf{h}=150 \mathrm{~km}$ in the ionosphere above EISCAT heating facility in Troms $\phi$, Norway.

The electron density profiles of the normal and heated ionosphere at height $\boldsymbol{h}=150 \mathbf{~} \mathbf{m}$ above EISCAT heater in Norway are also plotted vs. time and compared in Fig.7.

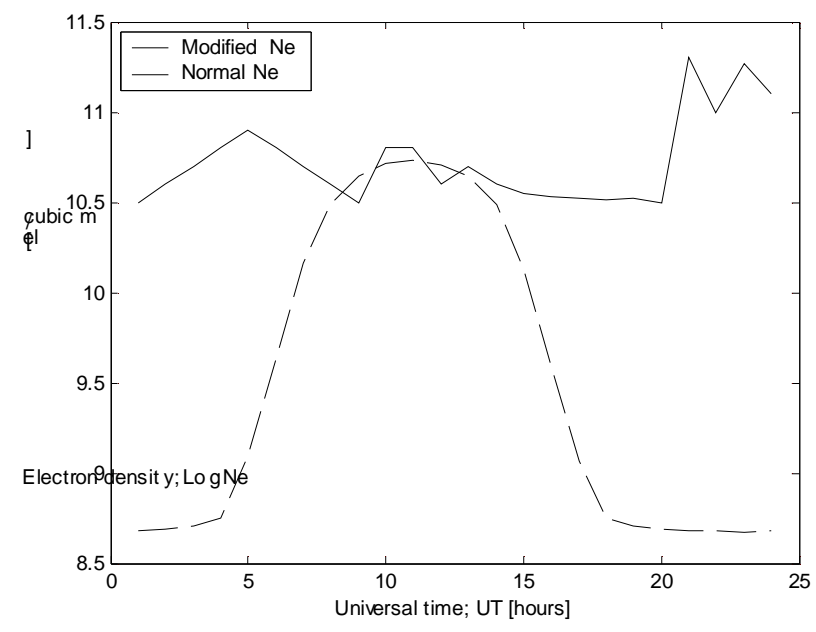

Figure(7): Variation of normal and modified electron density $\left(N_{e}\right)$ vs. universal time (UT) at $\boldsymbol{h}=150 \mathbf{~} \mathbf{m}$ in the ionosphere above EISCAT heating facility in Troms $\phi$, Norway.

Further inspection of Figures 6 and 7 discloses that the behavior of $\boldsymbol{T}_{i}, \boldsymbol{N}_{\boldsymbol{e}}$, and $\boldsymbol{T}_{\boldsymbol{e}}$ in later hours from 2230 UT was in much the same fashion as the changes of these parameters, which were observed from 2100 UT. One can see a very strong $\boldsymbol{T}_{\boldsymbol{e}}$ and $\boldsymbol{T}_{\boldsymbol{i}}$ increase (up to 
$1800 \mathrm{~K}$ ) closely correlated to the heater-on periods of 2100-2104 and 2220-2224 UT. Note that $\boldsymbol{T}_{\boldsymbol{e}}$ and $\boldsymbol{T}_{\boldsymbol{i}}$ values were about $700 \mathrm{~K}$ and $600 \mathrm{~K}$ just before the heater was turned on, as shown in Fig.6. The EISCAT heater-induced effects in the behavior of relative plasma partial pressure $\boldsymbol{P} / \boldsymbol{P}_{\boldsymbol{0}}$ were observed vs. time at $150 \mathrm{~km}$ altitude, and hence the disruption coefficient is calculated and plotted in Fig.8, which clearly shows that the hydrodynamical equilibrium has been disrupted due to the effects of powerful HF radio waves. The strongest increase in the disruption coefficient takes place in the heating cycles at 2100-2104 and 2220-2224 UT.

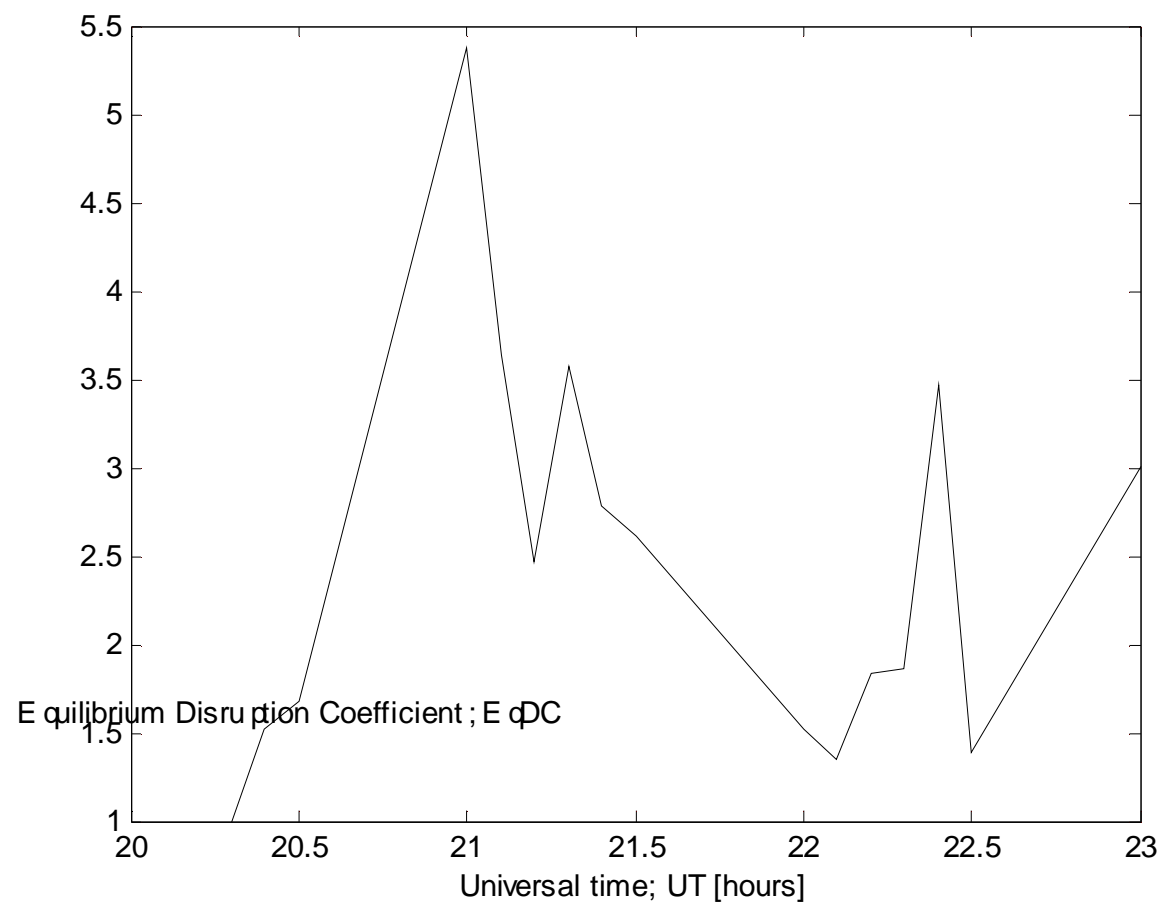

Figure(8): Variation of ionosphere equilibrium disruption coefficient (EqDC) vs. universal time (UT) at $\mathbf{h}=150 \mathrm{~km}$ in the ionosphere above EISCAT heating facility in Troms $\phi$.

Furthermore, it should be pointed out that the Troms $\varnothing$ dynasonde ionograms as well as the altitude temporal variations of the electron density measured by the EISCAT UHF radar in the course of the Troms $\varnothing$ pumping experiments on February 16 1996, show the presence of a most remarkable optical phenomenon during the two heating cycles of 2120-2124 and 2130-2134 UT. An intense auroral layer was observed near Troms $\emptyset$ after the heater was turned on. It is suggested that the reason for the initiation of artificial aurora during the Troms $\varnothing$ pumping experiments on February 161996 is due to the fulfillment of the plasma matching condition when the pump wave interacts strongly with the region containing the highest plasma density in the ionosphere. It is clear from Fig.9 that the calculated maximum plasma frequency $\left(\boldsymbol{F}_{\boldsymbol{p}}=\mathbf{4 . 0 4} \mathbf{M H z}\right)$ during the heating cycles of 2120-2124 and 2130-2134 UT is found to be equal to the pumping heater frequency. Thus the plasma matching condition is satisfied, and the resulting plasma resonances can enhance the local 
electromagnetic fields, and therefore their interactions with plasma particles leading to turbulences, local heating, density perturbations, and field aligned striations (FAS). Optical emissions and auroras are also initiated and enhanced under such conditions. The non-linear process at the resonance layer can stimulate emission of electromagnetic waves (SEE) which appear as the sidebands of the reflected EM waves [21-23].

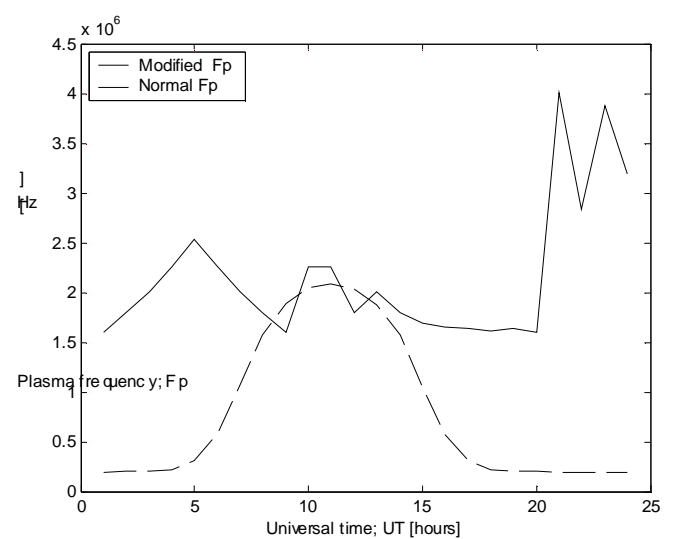

Figure(9): Variation of normal and Modified plasma frequency $\left(\boldsymbol{F}_{p}\right)$ vs. UT at $\mathbf{h}=\mathbf{1 5 0} \mathbf{~ k m}$ in the ionosphere above EISCAT heating facility in Troms $\phi$.

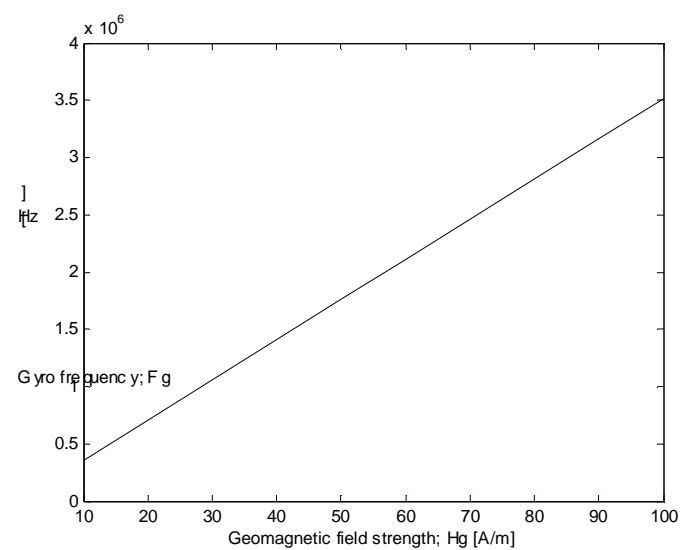

Figure(10): Electron cyclotron frequency $\left(\boldsymbol{F}_{g}\right)$ variation vs. geomagnetic field strength

It is important to discriminate between the plasma and upper hybrid (UH) matching conditions [24]. It is common knowledge that HF pump induced electron heating, as well as the excitation of artificial field-aligned irregularities (AFAI) and the anomalous absorption, is a phenomenon related to the upper hybrid wave [25]. It is generated at the vicinity of the upper hybrid resonance (UHR) altitude, where the heater frequency $\boldsymbol{F}_{\boldsymbol{h}}$ is equal to the local upper hybrid frequency; $\boldsymbol{F}_{\boldsymbol{h}}{ }^{2}=\boldsymbol{F}_{\boldsymbol{p}}{ }^{2}+\boldsymbol{F}_{g}{ }^{2}$, where $\boldsymbol{F}_{\boldsymbol{p}}$ is the local plasma frequency and $\boldsymbol{F}_{g}$ is the electron gyro frequency. From figures 9 and 10, the upper hybrid resonance is possible at cyclotron electron frequency $\boldsymbol{F}_{g}=\mathbf{0 . 5 6 6} \mathbf{M H z}$ realized at geomagnetic field strength $\mathrm{H}_{\mathrm{g}}=16$ $\mathrm{A} / \mathrm{m}$. Further, stronger pumping EM interaction with the ionosphere occurs when the pump frequency matches the maximum plasma frequency and also matches the second gyro harmonic frequency; i.e. $\boldsymbol{F}_{\boldsymbol{h}}=\boldsymbol{F}_{\boldsymbol{p}}=\mathbf{2} \boldsymbol{F}_{g}$, which is realized at $\boldsymbol{F}_{g}=\mathbf{2} \mathbf{M H z}$ and geomagnetic field strength $\boldsymbol{H}_{g}=\mathbf{5 6} \mathbf{A} / \mathbf{m}$. The previously matching modes (plasma, upper hybrid, and gyro harmonics) conditions are possible since the different geomagnetic field components span the range from $829 \boldsymbol{n T}$ up to $\mathbf{5 3 0 0 0} \boldsymbol{n T}$ as shown in Table 2. 
Table (2) Magnetic fields calculations in Troms $\phi$, Norway

\begin{tabular}{|c|c|c|c|c|c|c|c|}
\hline $\begin{array}{l}\text { Lat: } 6^{\circ} 39^{\prime} 36^{\prime \prime} \\
\text { Lon: } 18^{\circ} 57^{\prime} \\
\text { Elev: } 0.00 \mathrm{~m}\end{array}$ & $\frac{\text { Declination }}{+ \text { East }}$ & 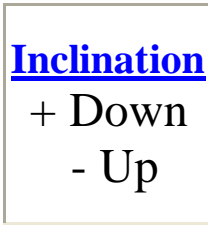 & $\frac{\text { Horizontal }}{\text { Intensity }}$ & 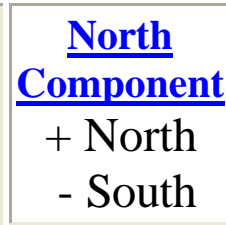 & $\frac{\begin{array}{c}\text { East } \\
\text { Component }\end{array}}{+ \text { East }}$ & $\frac{\frac{\text { Vertical }}{\text { Component }}}{+\begin{array}{c}\text { Down } \\
-U p\end{array}}$ & $\begin{array}{l}\text { Total } \\
\underline{\text { Field }}\end{array}$ \\
\hline 2/16/1996 & $4^{\circ} 19^{\prime}$ & $77^{\circ} 57^{\prime}$ & $\begin{array}{c}11,012.5 \\
\mathrm{nT}\end{array}$ & $10,981.2 \mathrm{nT}$ & $829.2 \mathrm{nT}$ & $51,578.3 \mathrm{nT}$ & $\begin{array}{c}52,740.9 \\
\text { nT }\end{array}$ \\
\hline $\begin{array}{c}\text { Change per } \\
\text { year }\end{array}$ & 12 per year & 1' per year & $\begin{array}{c}-11.7 \\
\text { nT/year }\end{array}$ & $\begin{array}{c}-14.5 \\
\text { nT/year }\end{array}$ & $\begin{array}{c}36.1 \\
\text { nT/year }\end{array}$ & $\begin{array}{c}31.4 \\
\text { nT/year }\end{array}$ & $\begin{array}{c}28.3 \\
\text { nT/year }\end{array}$ \\
\hline
\end{tabular}

\section{Conclusions:}

Experimental results concerning the possibility of an artificial modification of the ionosphere by HAARP and EISCAT HF heating facilities in Norway and USA have been presented, modeled, analyzed, discussed and compared. The proposed model has been used to analyze the ionospheric heating with pumping HAARP HF waves having frequency $\boldsymbol{f}=\mathbf{9} \mathbf{M H z}, X$-mode polarization, and effective radiated powers $\boldsymbol{E R P}=\mathbf{8 3 . 4}$ $\boldsymbol{d B W}$ and $95.7 \boldsymbol{d B W}$. The numerical results showed that severe disruption of the ionosphere hydrodynamical equilibrium due to the injected powerful EM waves in the ionosphere above HAARP heating facility at Alaska, USA. Under the unique conditions of matching the heater frequency $\mathbf{F}_{\mathbf{h}}=\mathbf{4 0 4 0} \mathbf{~ k H z}$ to the peak plasma frequency $\boldsymbol{F}_{\boldsymbol{p}}$, the heating effects of the $\boldsymbol{O}$-mode powerful EM wave with $\boldsymbol{E R P}=150 \boldsymbol{M W}$ pumped by EISCAT heater in Troms $\varnothing$, Norway, are strongly enhanced and amplified, This leads to strong wave activity and turbulence, local electron and ion heating, and modification of electron and ion density profiles. The resultant field-aligned striations (FAS) initiates a nonlinear feedback mechanism that leads to artificial aurora activation as observed on February 161996 above Troms $\varnothing$, Norway. The upper hybrid resonance (UHR) conditions to generate the upper hybrid waves (UHW) $\boldsymbol{F}_{\boldsymbol{h}}{ }^{2}=\boldsymbol{F}_{\boldsymbol{p}}{ }^{2}+\boldsymbol{F}_{g}{ }^{2}$, have also been investigated, and found to be realized at electron cyclotron frequency $\boldsymbol{F}_{g}=566 \mathbf{k H z}$ if the geomagnetic field strength $\left(\boldsymbol{H}_{g}\right)$ fluctuated down to $16 \mathrm{~A} / \mathrm{m}$. For higher $\boldsymbol{H}_{\boldsymbol{g}}$ up to 56 $\boldsymbol{A} / \boldsymbol{m}$, the second gyroharmonic frequency matches the heater frequency $\boldsymbol{F}_{\boldsymbol{h}}$ and the maximum plasma frequency $\boldsymbol{F}_{\boldsymbol{p}}$ leading to stronger nonlinear feedback mechanism. 
Proceedings of the $\boldsymbol{6}^{\text {th }}$ ICEENG Conference, 27-29 May, 2008

EE168 - 14

\section{References:}

[1] Davies, Kenneth, “Ionospheric Radio,” Peter Peregrinus Ltd.: London, 1990

[2] Hunsucker, R.D., Radio Techniques for Probing the Terrestrial Ionosphere, Springer-Verlag:Berlin, 1991.

[3] Blagoveshchenskaya, N. F., V. A. Kornienko, A. V. Petlenko, A. Brekke, and M. T. Rietveld, "Geophysical phenomena during an ionospheric modification experiment at Tromsф,” Ann. Geophys., 16, 1212-1225, 1998.

[4] N. F. Blagoveshchenskaya, T. D. Borisoval, V. A. Kornienkol, B. Thid'e, M. T. Rietveld, M. J. Kosch, and T. B"osinger, "Phenomena in the ionospheremagnetosphere system induced by injection of powerful HF radio waves into nightside auroral ionosphere," Annales Geophysicae 23: 87-100, 2005.

[5] Rietveld, M. T., H. Kopka, and P. Stubbe, "D region characteristics deduced from pulsed ionospheric heating under auroral electrojet conditions," J. Atmos. Terr. Phys., 49, 311, 1986

[6] Rietveld, M. T., M. J. Kosch, N. F. Blagoveshchenskaya, V. A. Kornienko, T. B. Leyser, and T. K. Yeoman, "Ionospheric electron heating, optical emissions, and striations induced by powerful HF radio waves at high latitudes: Aspect angle dependence," J. Geophys. Res., 108(A4), 1141, doi:10.1029/2002JA009543, 2003.

[7] L. M. Duncan and W. E. Gordon. Ionospheric modification by high power radio waves. J. Atmos. Terr. Phys., 44:1009-1017, 1982.

[8] L. M. Erukhimov, S. A. Metelev, E. N. Myasnikov, N. A. Mityakov, and V. L. Frolov, "Artificial ionospheric turbulence (review)," Radiophys. Quantum Electron. Engl. Transl., 30:156-171, 1987.

[9] K. G. Budden. The propagation of radio waves. Cambridge University Press, London, 1985

[10] K. Davies. Ionospheric Radio Waves. Blaisdell Publishing Company, 1969.

[11] L. H. Brace and R. F. Theis, "An Empirical Model of the Interrelationship of Electron Temperature and Density in the Daytime Thermosphere at Solar Minimum,"Geophys. Res. Lett. 5, 275, 1978 
[12] D. Bilitza, L. H. Brace, and R. F. Theis, "Modeling of Ionospheric Temperature Profiles,” Adv. Space Res. 5, \#7, 53, 1985.

[13] D. N. Anderson, M. Mendillo, and B. Herniter, "A Semi-Empirical Low-Latitude Ionospheric Model,” Radio Sci. 22, 292, 1987.

[14] D. Bilitza, “International reference ionosphere 2000,” Radio Science 36, 261, 2001.

[15] Bernhardt, P. A., L. M. Duncan, and C. A. Tepley, "Artificial airglow excited by high-power radio waves,” Science, 242, 1022-1027, 1988.

[16] Boström, R., “A model of the auroral electrojets,” J. Geophys. Res.,69, 49834999, 1964.

[17] J. A. Fejer. "Ionospheric modification and parametric instabilities," Reviews of Geophysics and Space Physics, 17(1):135-53, 1979.

[18] A. V. Streltsov, G. M. Milikh and W. Lotko, "Simulation of ULF field-aligned currents generated by HF heating of the ionosphere," Journal of Geophysical Research, vol. 110, A04216, doi: 10.1029/2004JA010629, pp.1:11, 2005.

[19] N. F. Blagoveshchenskaya, T. D. Borisova, V. A. Kornienko, B. Thid'e, M. T. Rietveld, M. J. Kosch, and T. B"osinger, "Phenomena in the ionospheremagnetosphere system induced by injection of powerful HF radio waves into nightside auroral ionosphere," Annales Geophysicae 23: 87-100, 2005.

[20] N. F. Blagoveshchenskaya, V. A. Kornienkol, A. V. Petlenko, A. Brekke, M. T. Rietveld, " Geophysical phenomena during an ionospheric modification experiment at Tromsù, Norway,” Ann. Geophysicae 16, $1212 \pm 1225$ (1998)

[21] Fejer, j. a., "Ionospheric modification and parametric instabilities," Rev. Geophys., 17, 135-153, 1979.

[22] T. B. Leyser, "Stimulated electromagnetic emissions by high-frequency electromagnetic pumping of the ionospheric plasma," Space Sci. Rev., 98 (3-4): 223-228, 2001. 
[23] E. D. Tereshchenko, B. Z. Khudukon, M. T. Rietveld, B. Isham, T. Hagfors, and A. Brekke, "The relationship between small-scale and large-scale ionospheric electron density irregularities generated by powerful HF electromagnetic waves at high latitudes," Annales Geophysicae, 24, 2901-2909, 2006.

[24] T. B. Leyser, "Parametric interaction between upper hybrid and lower hybrid waves in heating experiments," Geophys. Res. LETT., 18: 408-411, 1991.

[25] T. B. Leyser, B. Thide, H. Derblom, A. Hedberg, and B. Lundborg, "Stimulated electromagnetic emissions near electron cyclotron harmonics in the ionosphere," PHYS. Rev. Lett., 63 (11): 1145-1147, 1989. 